\title{
Robust Positively Invariant Cylinders in Constrained Variable Structure Control
}

\author{
Hanz Richter \\ Cleveland State University, h.richter@csuohio.edu \\ Brian D. O'Dell \\ Nomadics, Inc. \\ Eduardo A. Misawa \\ Oklahoma State University
}

Follow this and additional works at: https://engagedscholarship.csuohio.edu/enme_facpub

Part of the Mechanical Engineering Commons

How does access to this work benefit you? Let us know!

\section{Publisher's Statement}

(C) 2007 IEEE. Personal use of this material is permitted. Permission from IEEE must be obtained for all other users, including reprinting/ republishing this material for advertising or promotional purposes, creating new collective works for resale or redistribution to servers or lists, or reuse of any copyrighted components of this work in other works.

\section{Original Citation}

Richter, H., O'Dell, B. D., and Misawa, E. A., 2007, "Robust Positively Invariant Cylinders in Constrained Variable Structure Control," Automatic Control, IEEE Transactions on, 52(11) pp. 2058-2069.

This Article is brought to you for free and open access by the Mechanical Engineering Department at EngagedScholarship@CSU. It has been accepted for inclusion in Mechanical Engineering Faculty Publications by an authorized administrator of EngagedScholarship@CSU. For more information, please contact library.es@csuohio.edu. 


\title{
Robust Positively Invariant Cylinders in Constrained Variable Structure Control
}

\author{
Hanz Richter, Associate Member, IEEE, Brian D. O'Dell, and Eduardo A. Misawa, Senior Member, IEEE
}

\begin{abstract}
This paper proposes the use of cylinders as primary invariant sets to be used in certain state-constrained control designs. Following the idea originally introduced by O'Dell, the primary invariant set is intersected with the state constraints to yield sets which retain the invariance under some conditions. Although several results presented here apply to fairly general nonlinear systems and primary invariant sets of any shape, the focus is on constrained sliding-mode control (SMC) using infinite cylinders as the primary invariant set. Their use is motivated by a coordinate transformation where the sliding motion is decoupled from the overall convergence to the origin. Robust positive invariance conditions are given for cylinders having convex and compact cross sections. For the case of cylinders with ellipsoidal cross sections, the invariance condition is given in the form of a linear matrix inequality. Further, a decision procedure to qualify each state constraint is given as a tool for the selection of the switching gain. A numerical example for a third-order plant illustrates the method.
\end{abstract}

Index Terms-Constrained control, positively invariant sets, sliding-mode control, variable structure control.

\section{INTRODUCTION}

$\mathbf{P}$ RACTICAL application of any control scheme usually involves a limited amount of control effort and physical constraints on some or all state variables. Variable structure techniques, in particular, sliding-mode control (SMC), have received widespread attention due to their trademark total disturbance rejection properties. In contrast with the vast amount of literature concerning the theory and design of SMC [1]-[6], published material on constrained SMC is scarce. On the other hand, literature on constrained linear control using set invariance concepts is again abundant. A survey of invariance theory, including a comprehensive list of references, is found in [7]. The purpose of this paper is to summarize our findings in applying set invariance concepts to the constrained SMC design problem. Unlike many works dealing with set invariance, we do not attempt to determine or approximate the largest invariant set for a particular constrained system, for this may be achieved with an allowable control that does not satisfy other design requirements. Instead, we assume that the sliding manifold has already been selected to meet nominal performance requirements for the sliding mode. The design is completed by selecting a switching gain that is appropriate for the intended constraints and allowable disturbance. Among the shapes selected for invariant sets, polyhedra offer good accuracy in expense of complexity, while ellipsoids are, in that sense, the opposite [7]. Semiellipsoidal sets were introduced by O'Dell and Misawa [8] as a compromise solution between polyhedral and ellipsoidal sets for linear systems under linear state feedback. A semiellipsoidal set is obtained by intersecting an invariant ellipsoid with the state constraints. In this paper, we take the same approach, but substitute the ellipsoid by an infinite cylinder whose axis represents, in some coordinate system, the distance to the sliding hyperplane. Conditions are given so that the constrained cylinder is positively invariant regardless of the disturbance; that is, the constrained cylinder is a robust positively invariant (RPI) set. Additional conditions are given for the intersection of the cylinder and the constraints to be again RPI. The intersection, to be called "constrained cylinder," is used at the design stage as a recoverable set, that is, the set of states from which the system can be started without constraint violation. Although only regulation to the origin is directly treated, it is known that many tracking problems reduce to the regulation of the error. Alternative approaches to constrained SMC are found, for instance, in [9] and references therein, where constraint satisfaction in the presence of uncertainty is achieved for a robotic manipulator by introducing penalties in a control Lyapunov function, and in [10], where disturbance is not considered and the control law is allowed to depend on initial conditions. This paper is organized as follows: Section II introduces basic definitions and results concerning robust positive invariance; Section III develops a general result concerning the invariance of the intersection of a primary RPI set of general shape with the linear state constraints; Section IV offers an overview of SMC in the context of our work and develops the central results for cylinders of arbritrary cross sections; Section V specializes the results to ellipsoidal cross sections and describes the LMI and critical switching gain methods; Section VI addresses the use of a boundary layer to avoid chattering, giving additional conditions to retain the validity of the original design; Section VII is a numerical example for a third-order plant; Section VIII describes some possible extensions to the work; and Section IX offers conclusions.

\section{BASIC THEORY OF RoBUst Positive InVARIANCE}

Although the systems which this paper addresses are linear, the results of this section apply to more general systems, possibly time varying. Given a dynamic system described by $\dot{x}=$ $f(x(t))$, having a unique solution $x(t)$ in a subset $\Omega \subset \mathbb{R}^{n}$, the set $\mathcal{S} \subset \Omega$ is said to be positively invariant (PI) for the system if, for every initial state $x_{o} \in \mathcal{S}$, the solution $x(t)$ belongs to $\mathcal{S}$ 
for $t>0$. For the uncertain system $\dot{x}=f(x(t), \omega(t))$, where $\omega(t)$ is a function known to have values in some set $\mathcal{W}$, the set $\mathcal{S}$ is said to be RPI with respect to $\mathcal{W}$ if for all $x_{o} \in \mathcal{S}$ and for all functions $\omega$ the solution $x(t)$ belongs to $\mathcal{S}$ for $t>0$. The definition does not restrict the class of functions to which $\omega$ belongs, as long as a unique solution to $\dot{x}=f(x(t), \omega(t))$ exists for all initial conditions in $\Omega$. The widely known result due to Nagumo [11] provides a necessary and sufficient condition for the invariance of a set $\mathcal{S}$ in terms of its tangent cone, also known as Bouligand contingent cone. A precise definition of tangent cone is given, for instance, in [7]. For our purposes, it will suffice to recall the tangent cones of particular sets, and to cite some relevant properties. The notation $\mathcal{C}_{\mathcal{S}}(x)$ will be used for the tangent cone of set $\mathcal{S}$ at a point $x \in \mathbb{R}^{n}$. The notations $A^{c}, \bar{A}, \partial A$, and $A \subset B$ indicate, as usual, the complement, closure and boundary of $A$, and that $A$ is a subset (not necessarily proper) of $B$, respectively. The interior of a set $A$ is denoted as $\operatorname{int}(A)$. The extended real line is denoted here as $\mathbb{R}^{*}=\mathbb{R} \cup\{\infty\} \cup\{-\infty\}$.

Theorem 1 [11]: Consider the system $\dot{x}=f(x(t))$ having a unique solution for each initial condition in a set $\Omega$. Let $\mathcal{S} \subset \Omega$ be a closed and convex set. Then, $\mathcal{S}$ is PI for the system if and only if $f(x) \in \mathcal{C}_{\mathcal{S}}(x)$ for all $x \in \partial \mathcal{S}$. Mechanically interpreted, the theorem formalizes the intuitive notion of invariance being attained when the velocity vectors $\dot{x}$ at the boundary of the set all point into or are tangent to $\mathcal{S}$. The following important extension of Nagumo's result is given in [7] and concerns robust positive invariance.

Theorem 2 [7]: Consider the uncertain system $\dot{x}=$ $f(x(t), \omega(t))$ where the uncertain input $\omega(t)$ has values in $\mathcal{W}$ for all $t \geq 0$. Assume that the system possesses a unique solution for all initial conditions $x_{0} \in \Omega$ and all $\omega(t)$. Then, the convex and closed set $\mathcal{S} \subset \Omega$ is RPI with respect to (w.r.t.) $\mathcal{W}$ if and only if $f(x, \omega) \in \mathcal{C}_{\mathcal{S}}(x)$ for all $x \in \partial \mathcal{S}$ and for all $\omega \in \mathcal{W}$.

Note that the RPI property depends only on the range of values taken by $\omega(t)$. This allows one to speak of the RPI of $\mathcal{S}$ with respect to $\mathcal{W}$. A few useful properties of tangent cones are now stated.

Property 1 [12], [13]: If $A$ and $B$ are closed and convex sets such that $0 \in \operatorname{int}(A-B)$, then $\mathcal{C}_{A \cap B}(x)=\mathcal{C}_{A}(x) \cap \mathcal{C}_{B}(x)$ for all $x \in A \cap B$.

In the previous property and throughout this paper, the notation $A \pm B$ is used for the set of the $x \pm y$ such that $x \in A$ and $y \in B$, where $A$ and $B$ are subsets of $\mathbb{R}^{n}$. The set-theoretic difference, in contrast, is denoted as $A \cap B^{c}$.

Property 2 [12], [7]: If $A$ is convex, then $\mathcal{C}_{A}(x)$ is convex for all $x \in A$.

It is also a fact [12], [7] that if $x \in \operatorname{int}(A)$ then $\mathcal{C}_{A}(x)=\mathbb{R}^{n}$. To end this section, we show that, for a certain class of systems, and when the uncertain input belongs to a closed interval, it is sufficient to check for positive invariance of the system at extreme values of the input.

Theorem 3: Consider a system that is linear in the uncertainty, e.g., $\dot{x}=f(x(t), \omega(t))=g(x(t))+B \omega(t)$ where $\omega(t) \in$ $\mathcal{I}=[\underline{\omega}, \bar{\omega}] \forall t \geq 0$. Assume that the system possesses a unique solution for all initial conditions $x_{0} \in \Omega$ and all $\omega(t) \in \mathcal{I}$. Then, $\mathcal{S} \subset \Omega$ is RPI w.r.t. $\mathcal{I}$ if and only if it is PI for $\dot{x}=f(x(t), \bar{\omega})$ and for $\dot{x}=f(x(t), \bar{\omega})$.
Proof: Suppose $\mathcal{S}$ is positively invariant for $\dot{x}=$ $f(x(t), \bar{\omega})$ and for $f(x(t), \bar{\omega})$. Then, by Theorem 1 , $f(x, \bar{\omega}) \in \mathcal{C}_{\mathcal{S}}(x)$ and $f(x, \bar{\omega}) \in \mathcal{C}_{\mathcal{S}}(x)$ for all $x \in \partial \mathcal{S}$. Let $\omega \in \mathcal{I}$. Then, $\omega=\alpha \bar{\omega}+(1-\alpha) \underline{\omega}$ for some $\alpha \in[0,1]$ and thus $f(x, \omega)=\alpha f(x, \bar{\omega})+(1-\alpha) f(x, \bar{\omega})$. Since $\mathcal{C}_{\mathcal{S}}(x)$ is convex whenever $S$ is convex, we conclude that $f(x, \omega) \in \mathcal{C}_{\mathcal{S}}(x)$ for all $x \in \partial \mathcal{S}$ and for all $\omega \in \mathcal{I}$. By Theorem $2, \mathcal{S}$ is RPI. The reverse implication is trivial.

\section{State-Constrained InVARIANT SetS}

Commonly used state constraints are typically specified as a convex polyhedron. The use of the constraints themselves as RPI set is difficult to accomplish. For this reason, sets of simpler description are used as primary invariant sets. The set can be required, for instance, to be a subset of the state constraint set, thus guaranteeing that the latter will not be violated. This, however, can be conservative. Instead, we consider the possibility of intersecting the state constraints with the primary invariant set.

Definition 1: A linear state constraint set is defined as $G=$ $\cap G_{i}$ for $i=1,2 \cdot \cdot m$, where $G_{i}=\left\{w \in \mathbb{R}^{n}: \Gamma_{i} w \leq 1\right\} \cdot \Gamma_{i}$ are row vectors such that $G$ is a convex set containing the origin in its interior.

Note that $\partial G_{i}$, the boundary of $G_{i}$, is the set of points such that $\Gamma_{i} w=1$. At this point, it is convenient to recall [12], [7] that the tangent cone to a linear state constraint $\Gamma_{i} w \leq 1$ is given by the points $v$ such that $\Gamma_{i} v \leq 0$. In connection with the uncertain system $\dot{w}=f(w, \zeta(t))$, where $\zeta(t) \in \mathcal{Z}$ for all $t \geq 0$, introduce the following sets:

$$
\begin{aligned}
& G_{i}^{+}=\left\{w \in \mathbb{R}^{n}: \Gamma_{i} f(w, \zeta)>0 \text { for some } \zeta \in \mathcal{Z}\right\} \\
& G_{i}^{-}=\left\{w \in \mathbb{R}^{n}: \Gamma_{i} f(w, \zeta)<0 \text { for all } \zeta \in \mathcal{Z}\right\} .
\end{aligned}
$$

The next result concerns the invariance of the intersection of a state constraint set and an RPI set. It generalizes Theorem 1 from [8], in that the system is not restricted to be linear under state feedback, the positively invariant set does not have to be an ellipsoid, disturbance is allowed, and the constraints may be asymmetric.

Theorem 4: Consider the system $\dot{w}=f(w, \zeta(t))$, where $\zeta(t) \in \mathcal{Z}$ for $t \geq 0$ and let $M \subset \mathbb{R}^{n}$ be a compact and convex set which is RPI w.r.t. $\mathcal{Z}$. Let $G=\cap G_{i}, i=1,2 \cdot \cdot m$ be a linear state constraint set as in Definition 1. Assume, further, that $0 \in \operatorname{int}(M-G)$. Denote $\mathcal{S}=M \cap G$. Then, $\mathcal{S}$ is RPI w.r.t. $\mathcal{Z}$ if and only if $\mathcal{S} \cap \partial G_{i} \cap G_{i}^{+}=\varnothing$ for $i=1,2 \ldots m$.

The proof of Theorem 4 can be found in the Appendix.

\section{APPLiCATION TO SMC OF LINEAR SYSTEMS}

\section{A. Overview of $S M C$}

SMC is a widely studied technique [1], [4], [6] that achieves total insensitivity of the controlled variables to certain kinds of disturbances and parameter uncertainties. In this section, we briefly introduce the salient characteristics of linear systems under SMC. Consider the single-input, linear, and time-invariant system

$$
\dot{x}=A x+B u(t)+D \delta(t)
$$


where $A$ is an $n \times n$ matrix, $B$ and $D$ are column vectors, $x \in$ $\mathbb{R}^{n}, u$ is the scalar control input, and $\delta(t)$ is a scalar, unknown disturbance. It is assumed that the pair $(A, B)$ is controllable and that the matching condition

$$
[\operatorname{rank}[B \mid D]=\operatorname{rank}(B)]
$$

is satisfied, so that the system can be rewritten as

$$
\dot{x}=A x+B(u(t)+\zeta(t)) \text {. }
$$

Assume that $\delta(t)$ is bounded so that $\zeta(t) \in \mathcal{Z}$ for all $t \geq 0$, where $\mathcal{Z}=[-\bar{\zeta}, \bar{\zeta}], \bar{\zeta}>0$. In order to specify an SMC law with linear sliding manifold, let $T$ be a nonsingular matrix satisfying $T^{-1} B=\left[\begin{array}{lll|l}0 & 0 & \ldots\end{array}\right]^{T}$, and such that $A_{11}$ is a nonsingular $n-1 \times n-1$ matrix, where $A_{i j}$ for $i, j=1,2$ are the partition blocks of $T^{-1} A T$ with $A_{22}$ scalar. Such transformation corresponds to the well-known regular form used in the SMC literature, with the additional restriction of $A_{11}$ being invertible. A method to find such $T$ for any controllable pair $(A, B)$ is given in the Appendix. Define a coordinate transformation $x=T z$ and write the system equations in the new coordinates as

$$
\dot{z}=T^{-1} A T z+\left[0^{T} \mid 1\right]^{T}(u(t)+\zeta(t)) .
$$

Consider the sliding manifold $s=\mathcal{G} x=\mathcal{G} T z=\left[\mathcal{G}_{1} \mid \mathcal{G}_{2}\right] z$. Without loss of generality, consider that $\mathcal{G}_{2}=1$. The control law

$$
u(t)=-A_{21} z_{1}-A_{22} z_{2}-\mathcal{G}_{1}\left(A_{11} z_{1}+A_{12} z_{2}\right)-\eta \operatorname{sgn}(s)
$$

results in the closed-loop dynamics described by

$$
\begin{aligned}
& \dot{z}_{1}=A_{11} z_{1}+A_{12} z_{2} \\
& \dot{z}_{2}=-\mathcal{G}_{1} A_{11} z_{1}-\mathcal{G}_{1} A_{12} z_{2}-\eta \operatorname{sign}(s)+\zeta(t) \\
& 1 s=\mathcal{G}_{1} z_{1}+z_{2} .
\end{aligned}
$$

It can be easily shown that any choice of $\mathcal{G}$ under the previous constraints on $T$, and such that $\mathcal{G}_{2}=1$, results in $\mathcal{G} B=1$. It is likewise straightforward to show that the closed-loop dynamics in the original coordinates is described by

$$
\dot{x}=(I-B \mathcal{G}) A x-B \eta \operatorname{sign}(s)+B \zeta(t) .
$$

The function $V=s^{2}$ has a negative derivative if $\eta>\bar{\zeta}$, showing that the control law (4) results in the state reaching the plane $s=0$ in finite time and remaining there indefinitely despite the presence of the disturbance [1], [4], [6]. Evolution of the closedloop system (5) for $s=0$ is independent of the disturbance and described by the reduced dynamics

$$
\dot{z}_{1}=A_{w} z_{1}
$$

where

$$
A_{w}=A_{11}-A_{12} \mathcal{G}_{1} .
$$

Thus, a stable sliding mode is obtained by choosing $\mathcal{G}_{1}$ such that $A_{w}$ has eigenvalues with negative real parts ( $A_{w}$ is Hurwitz). It can be shown that the controllability of $(A, B)$ guarantees that the eigenvalues of $A_{w}$ may be freely placed using $\mathcal{G}_{1}$. The existence and uniqueness of a solution to (5), as well as the existence of a sliding mode, have been widely considered in the standard SMC literature [6], [14], [15]. For the remainder of this paper, it will be assumed that $A_{w}$ is Hurwitz, $\eta>\bar{\zeta}$, and that a unique solution to the closed-loop SMC differential (5) exists for every initial condition in $\mathbb{R}^{n}$.

\section{B. Decomposition of the Closed-Loop Dynamics}

The Lyapunov function $V=s^{2}$ induces an obvious family of invariant sets, namely, the sets $S_{\gamma}=\left\{z \in \mathbb{R}^{n}:|s(z)| \leq \gamma\right\}$ for $\gamma \geq 0$ are all positively invariant. These sets are "naturally" invariant for systems under SMC. A coordinate transformation is introduced here that decouples the motion towards $s=0$ from the overall convergence to the origin. This decomposition will suggest a cylindrical shape for positively invariant sets.

Lemma 1: There exists a coordinate transformation $x=J w$ with $J$ nonsingular in which the closed-loop dynamics (5) is expressed as

$$
\left[\begin{array}{c}
\dot{w}_{1} \\
\hline \dot{w}_{2}
\end{array}\right]=\left[\begin{array}{c|c}
A_{w} & 0 \\
\hline 0 & 0
\end{array}\right]\left[\frac{w_{1}}{w_{2}}\right]+\left[\frac{B_{1}}{B_{2}}\right](\eta \operatorname{sign}(s)-\zeta(t))
$$

where $s=\mathcal{G}_{w} w_{2},\left[B_{1} \mid B_{2}\right]^{T}=-J^{-1} B \triangleq B_{w}$, and $\mathcal{G}_{w}=$ $-\mathcal{G}_{1} A_{11}^{-1} A_{12}+1$. Moreover, $B_{2} \mathcal{G}_{w}=-1$.

The proof to Lemma 1 is done directly by specifying $J$. In [16], it is shown that $J=T K$ is a valid choice, where $K$ is the invertible matrix given by

$$
K=\left[\begin{array}{c|c}
I & -A_{11}^{-1} A_{12} \\
\hline-\mathcal{G}_{1} & 1
\end{array}\right] .
$$

Note that $s$ is proportional to the scalar $w_{2}$ and independent of $w_{1}$. An immediate observation is that an arbitrary (and possibly infinite) real interval containing zero is RPI for the dynamics of $w_{2}$. The result is formalized in the following.

Lemma 2: For any initial condition $\left[w_{10}^{T} \mid w_{20}\right]^{T} \in \mathbb{R}^{n}$, where $w_{20} \in[a, b]$, with $a, b \in \mathbb{R}^{*}$ and $0 \in[a, b]$, the trajectory $w(t)$ of the closed-loop system (8) is such that $w_{2}(t) \in[a, b]$ for all $t>0$; that is, $[a, b]$ is RPI for the dynamics of $w_{2}$.

Lemma 2 follows directly from Lemma 1 by using the conditions $B_{2}\left(-\mathcal{G}_{1} A_{11}^{-1} A_{12}+1\right)=-1<0$ and $\eta>\bar{\zeta}$ in establishing the monotonic decrease of $|s|$ (and that of $\left|w_{2}\right|$ ) at both sides of $s=0$. With the aid of Lemma 2, it is now possible to specify cylindrical positive invariant sets with fairly general cross sections. Introduce the notation

$$
\mathcal{H}_{\mathcal{S}_{o}}[a, b]=\left\{\left[w_{1}^{T} \mid w_{2}\right]^{T} \in \mathbb{R}^{n}: w_{1} \in \mathcal{S}_{o}, w_{2} \in[a, b]\right\} .
$$

The set $\mathcal{S}_{O}$ is termed the cylinder's cross section. The following result follows directly from Lemma 2.

Theorem 5: Let $\mathcal{S}_{O} \subset \mathbb{R}^{n-1}$ be a compact and convex set containing the origin. Suppose $\mathcal{S}_{o}$ is RPI for the system $\dot{w}_{1}=$ $A_{w} w_{1}+B_{1} \zeta^{\prime}(t)$, where $A_{w}$ is Hurwitz and $\left|\zeta^{\prime}(t)\right| \leq \eta+\bar{\zeta}$ for 
all $t \geq 0$. Then, all cylinders $\mathcal{H}_{\mathcal{S}_{o}}[a, b]$ such that $0 \in[a, b]$ are RPI for the closed-loop dynamics of (8).

From now on, a compact and convex cross section will be assumed in the notation for cylinders. Also, the notation $\mathcal{H}_{\mathcal{S}_{o}}$ is used for the family of cylinders $\mathcal{H}_{\mathcal{S}_{o}}=\left\{\mathcal{H}_{\mathcal{S}_{o}}[a, b]: 0 \in[a, b], a, b \in \mathbb{R}^{*}\right\}$. We assume that $G$ is the constraint set in $w$-coordinates. That is, to each linear constraint $\Gamma_{i x} x \leq 1$ for the original coordinates, there corresponds a constraint $\Gamma_{i x} J w=\Gamma_{i} w \leq 1$. Since $J$ is nonsingular, it is straightforward to see that $G$ is convex if and only if the original constraint set in $x$-coordinates is so. For the remainder of this paper, assume that $\Gamma_{i x} B \neq 0$ for $i=1,2 \ldots m$. This is a basic problem feasibility assumption discussed, for instance, in [10]. Note that it implies that $\Gamma_{i} B_{w} \neq 0$ for $i=1,2 \ldots m$. Since $G$ is compact, the following quantities are well defined:

$$
\begin{aligned}
& \bar{w}_{2}=\max _{w \in G} w_{2} \\
& \underline{w}_{2}=\min _{w \in G} w_{2} .
\end{aligned}
$$

Note that $\underline{w}_{2}<0<\bar{w}_{2}$, since $0 \in \operatorname{int}(G)$. Now, a few auxiliary results are introduced concerning the properties of the sets defined in (1) and their boundaries, for the specific $f(w, \zeta)$ arising in the SMC closed-loop dynamics

$$
\begin{aligned}
f(w, \zeta) \triangleq & {\left[\left(A_{w} w_{1}+B_{1}(\eta \operatorname{sign}\right.\right.} \\
& \left.\left.\left.\times\left(\mathcal{G}_{w} w_{2}\right)-\zeta\right)\right)^{T} \mid B_{2}\left(\eta \operatorname{sign}\left(\mathcal{G}_{w} w_{2}\right)-\zeta\right)\right]^{T} .
\end{aligned}
$$

Equation (11) defines $f(w, \zeta)$ as the value of $\dot{w}$ in the SMC closed-loop dynamics for a constant disturbance value. The boundary of $G_{i}^{+}$separates $\mathbb{R}^{n}$ into two regions $\operatorname{int}\left(G_{i}^{+}\right)$and $\operatorname{int}\left(G_{i}^{-}\right)$where the value of $\Gamma_{i} w$ increases (constraint violation) and decreases (constraint satisfaction), respectively. The following result precisely states this observation and gives an explicit formula for $\partial G_{i}^{+}$.

Proposition 1: Suppose $\Gamma_{i}=\left[\Gamma_{i 1} \mid \Gamma_{i 2}\right]$, where $\Gamma_{i 1} A_{w} \neq 0$. Then, the collection of subsets $\left\{\operatorname{int}\left(G_{i}^{+}\right), \operatorname{int}\left(G_{i}^{-}\right), \partial G_{i}^{+}\right\}$corresponding to $f(w, \zeta)$ of (11) is a partition of $\mathbb{R}^{n}$. Furthermore

$$
\begin{aligned}
& \partial G_{i}^{+}=\left\{w \in \mathbb{R}^{n}: \Gamma_{i} f\left(w, \zeta^{*}\right)=0, w_{2} \neq 0\right\} \cup \\
& \quad\left\{\left[w_{1}^{T} \mid 0\right]^{T} \in \mathbb{R}^{n}:\left|\Gamma_{i 1} A_{w} w_{1}-\Gamma_{i} B_{w} \zeta^{*}\right| \leq\left|\Gamma_{i} B_{w}\right| \eta\right\}
\end{aligned}
$$

where $\zeta^{*}=-\operatorname{sign}\left(\Gamma_{i} B_{w}\right) \bar{\zeta}$.

The shape of the set $\partial G_{i}^{+}$and the partition it induces is schematically depicted in Fig. 1 for $n=3$. The boundary $\partial G_{i}^{+}$, labeled with zeros, is composed of two "vertical" half-planes situated at opposite sides of $w_{2}=0$, together with the line $\Gamma_{i 1} A_{w} w_{1}=0$ at $w_{2}=0$. The regions indicated with plus and minus signs correspond to $G_{i}^{+}$and $G_{i}^{-}$, respectively. The constraint shown would not satisfy Corollary 1 , since it intersects $\partial G_{i}^{+}$inside the cylinder.

The second result establishes the existence of at least one viable point on the intersection of the constraint boundary and the interior of an RPI cylinder. That is, it discards the possibility that all points on the portion of the boundary inside the RPI cylinder result in constraint violation.

Lemma 3: Let the family of cylinders $\mathcal{H}_{\mathcal{S}_{o}}$ be RPI for the SMC dynamics (8). Let a linear state constraint $G_{i}$ be such that

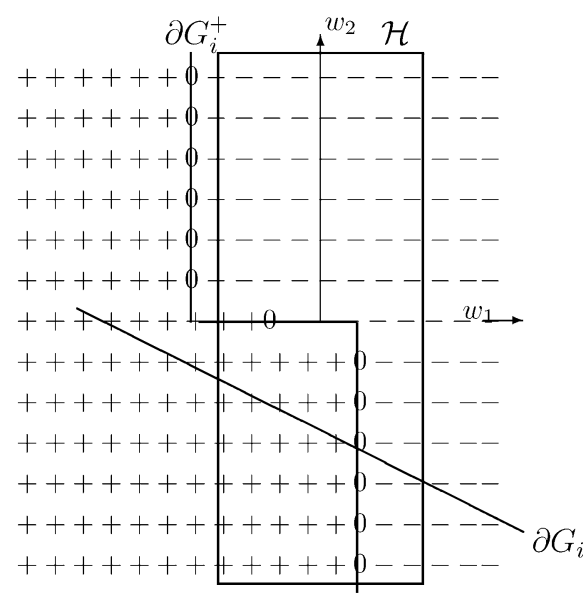

Fig. 1. Set $\partial G_{i}^{+}$and the partition it induces.

$\partial G_{i} \cap \mathcal{H}_{\mathcal{S}_{o}}\left[\underline{w}_{2}, \bar{w}_{2}\right] \neq \varnothing$. Then, $\exists w \in \mathcal{H}_{\mathcal{S}_{o}}\left[\underline{w}_{2}, \bar{w}_{2}\right] \cap \partial G_{i}$ such that $\Gamma_{i} f(w, \zeta)<0 \forall \zeta \in \mathcal{Z}$.

The proof of Lemma 3 is shown in the Appendix, while the proof of the more intuitive Proposition 1 has been omitted due to space limitations. Interested readers are referred to [16] for details.

The following corollary to Theorem 4 provides a sufficient condition for RPI that leads to computation.

Corollary 1: Let the family of cylinders $\mathcal{H}_{\mathcal{S}_{o}}$ be RPI for the SMC dynamics (8) and let $H \in \mathcal{H}_{\mathcal{S}_{o}}$. Let $G$ be a constraint set such that $0 \in \operatorname{int}(G-H)$. Then, $\mathcal{S}=H \cap G$ is RPI for (8) if $\mathcal{H}_{\mathcal{S}_{o}}\left[\underline{w}_{2}, \bar{w}_{2}\right] \cap \partial G_{i} \cap \partial G_{i}^{+}=\varnothing \forall i=1,2 \ldots m$.

Corollary 1 is proven with the aid of Lemma 3 and Proposition 1. The proof appears in the Appendix.

Comments on Conservativeness: While Theorem 4 is a necessary and sufficient condition, its use would require the exploration of the whole $G_{i}^{+}$region. As done in [8] and [17], computational tractability is obtained by expressing the result in terms of the boundary $\partial G_{i}^{+}$, a set of reduced dimensionality. In doing so, the condition becomes only sufficient. However, consider a set $\mathcal{S}$ which is RPI but fails the condition of the Corollary. It can be seen that this can only occur for points $w \in \partial G_{i} \cap \partial G_{i}^{+}$ belonging to the boundary of the cylinder. Therefore, a new RPI cylinder which passes the condition can be obtained by an infinitesimally small reduction in volume. This implies that the result, for all practical purposes, is not conservative.

\section{Cylinders With Ellipsoidal Cross Sections}

The results presented previously apply to a fairly large class of cylinder cross sections $\mathcal{S}_{o}$. Ellipsoidal invariant sets are extensively used in computations due to the correspondence to quadratic Lyapunov functions and their simplicity in relation to the also commonly used polyhedral sets. Ellipsoidal sets, however, can be conservative. The semiellipsoidal sets introduced by O'Dell for linear systems under state feedback achieve a good compromise between simplicity and conservativeness. A semiellipsoidal set is obtained by intersecting a linear state constraint set and an ellipsoidal invariant set [8], [17]. In this section, we restrict $\mathcal{S}_{O}$ to be ellipsoidal and develop results leading to design calculations for constrained SMC. As seen in the previous sections, a cylinder is first required to be RPI for it to yield 
an RPI set upon intersection with the state constraints. By Theorem 5, we see that the cylinder cross section is required to be itself RPI for a linear system driven by a bounded disturbance. Moreover, by Theorem 3, it is sufficient to establish invariance for extreme disturbance values.

\section{A. RPI Cylinders}

Consider the system

$$
\dot{w}_{1}=A_{w} w_{1}+B_{1} \zeta^{\prime}(t)
$$

where $\zeta^{\prime} \in\left[-\bar{\zeta}^{\prime}, \bar{\zeta}^{\prime}\right]$. We wish to find conditions under which the ellipsoidal set $\mathcal{E}=\left\{w_{1} \in \mathbb{R}^{n-1}: w_{1}^{T} P w_{1} \leq 1\right\}$ with $P>0$ is RPI for the dynamics (12). To this effect, note that [7] the tangent cone of $\mathcal{E}$ at its boundary $\partial \mathcal{E}$ is given by $\mathcal{C}_{\mathcal{E}}\left(w_{1}\right)=$ $\left\{y: 2 w_{1}^{T} P y \leq 0\right\}$. Nagumo's condition results in

$$
2 w_{1}^{T} P\left(A_{w} w_{1}+B_{1} \zeta^{\prime}(t)\right) \leq 0 \text { along } w_{1}^{T} P w_{1}=1
$$

Conditions on $P$ for (13) to hold can be derived following two approaches. One involves linear matrix inequalities (LMIs) and lends itself to ellipsoid volume optimization. The other approach has a simpler form and provides the maximum sum of disturbance bound and switching gain allowable for a particular ellipsoid.

\section{B. LMI Approach}

Nagumo's condition (13) is equivalent to a quadratic boundedness requirement, which, as shown in [18], can be equivalently expressed by the LMI

$$
\left[\begin{array}{cc}
P A_{w}+A_{w}^{T} P+\alpha P & \overline{\zeta^{\prime}} P B_{1} \\
\bar{\zeta}^{\prime} B_{1}^{T} P & -\alpha I
\end{array}\right] \leq 0
$$

where $P=P^{T}>0$ is sought.

Lemma 4: The set $\mathcal{E}=\left\{w_{1} \in \mathbb{R}^{n}: w_{1}^{T} P w_{1} \leq 1\right\}$ is RPI for system (12) if there exist a symmetric, positive-definite matrix $P$ and a scalar $\alpha>0$ such that the LMI (14) holds.

Note that LMI (14) is always feasible, since $A_{w}$ is Hurwitz. Moreover, only values of $\alpha$ less than the maximum decay rate need to be considered, that is, $\alpha$ satisfies $0<\alpha<2|\operatorname{Re}(\bar{\lambda})|$, where $\bar{\lambda}$ is that eigenvalue of $A_{w}$ with the largest real part in absolute value [18]. The previous matrix inequality can be readily solved using, for instance, the Matlab LMI toolbox.

\section{Critical Switching Gain}

A computationally simpler alternative is obtained by using Theorem 3. Positive invariance is now required for the two autonomous systems that result when either $-\bar{\zeta}^{\prime}$ or $\bar{\zeta}^{\prime}$ are used. In view of the symmetry of $P$, Nagumo's condition can be restated as

$$
\begin{array}{r}
w_{1}^{T}\left(A_{w}^{T} P+P A_{w}\right) w_{1} \pm 2 \bar{\zeta}^{\prime} B_{1}^{T} P w_{1} \leq 0 \\
\text { along } w_{1}^{T} P w_{1}=1
\end{array}
$$

Define

$$
A_{w}^{T} P+P A_{w}=-Q
$$

It is straightforward to prove that a necessary condition for (15) to hold for some $P>0$ is that $Q>0$. Thus, we may assume that $P$ is the unique symmetric, positive-definite solution to the previous Lyapunov equation for arbitrary $Q>0$, guaranteed to exist due to $A_{w}$ being Hurwitz. Given $Q=Q^{T}>0$ and $P=P^{T}>0$, there exists some $\beta>0$ such that $w_{1}^{T}\left(A_{w}^{T} P+\right.$ $\left.P A_{w}\right) w_{1}=-w_{1}^{T} Q w_{1} \leq-\beta w_{1}^{T} P w_{1}$ for all $w_{1} \in \partial \mathcal{E}$. The quantity $\beta$ is bounded by $\bar{\beta}=\min \left\{w_{1}^{T} Q w_{1}: w_{1} \in \partial \mathcal{E}\right\}$. The bound $\bar{\beta}$ is readily obtained by Lagrange multipliers and given by

$$
\bar{\beta}=\frac{v^{T} Q v}{\gamma^{2}}
$$

where $\gamma^{2}=v^{T} P v$ and $v$ is that eigenvector of $P^{-1} Q$ yielding the least value for the right-hand side of (16). Knowing $\bar{\beta}$, it now follows that inequality (15) holds in $\partial \mathcal{E}$ if $\pm 2 \bar{\zeta}^{\prime} B_{1}^{T} P w_{1} \leq \bar{\beta}$. Moreover, since $\bar{\beta}>0$, we consider

$$
\begin{aligned}
2 \overline{\zeta^{\prime}}\left|B_{1}^{T} P w_{1}\right| & \leq \bar{\beta} \\
\text { along } w_{1}^{T} P w_{1} & =1 .
\end{aligned}
$$

To obtain a condition equivalent to (17), we solve

$$
\begin{aligned}
& \max 2 \bar{\zeta}^{\prime}\left|B_{1}^{T} P w_{1}\right| \quad \text { s.t. } \\
& w_{1}^{T} P w_{1}=1 .
\end{aligned}
$$

This is readily solved using Lagrange multipliers. The maximum is $2 \bar{\zeta}^{\prime} \sqrt{B_{1}^{T} P B_{1}}$, which must be less than $\bar{\beta}$. The resulting invariance condition can be also shown to be necessary, and it is summarized in the following.

Lemma 5: The set $\mathcal{E}=\left\{w_{1} \in \mathbb{R}^{n}: w_{1}^{T} P w_{1} \leq 1\right\}$ is RPI for system (12) if and only if there exist symmetric, positive-definite matrices $P$ and $Q$ such that

$$
A_{w}^{T} P+P A_{w}=-Q
$$

and

$$
\bar{\zeta}^{\prime} \leq \frac{\bar{\beta}}{2 \sqrt{B_{1}^{T} P B_{1}}}
$$

with $\bar{\beta}$ defined in (16).

Lemmas 5 and 4 lead to computation of RPI cylinders for SMC without regard to constraints. Summarizing, the ellipsoidal cross section is made itself RPI by either the LMI or critical switching gain methods, taking into account $\bar{\zeta}^{\prime}=\eta+\bar{\zeta}$ and $\eta>\bar{\zeta}$. Theorem 5 guarantees that all cylinders of the family $\mathcal{H}_{\mathcal{E}}$ are RPI.

\section{State-Constrained RPI Cylinders}

In this section, we apply Corollary 1 to cylinders with ellipsoidal cross sections and provide results leading to design calculations. One way to satisfy the condition in the Corollary is to find the point $w^{*}$ of $\partial G_{i}^{+} \cap \partial G_{i}$ (if any) for which $w_{1}^{T} P w_{1}$ is 
minimum and enforce $w_{1}^{T *} P w_{1}^{*}>1$. In other words, we wish to solve the optimization problem

$$
\begin{aligned}
& \min _{w_{2} \in \mathbb{R}} w_{1}^{T} P w_{1} \quad \text { s.t. } \\
& {\left[w_{1}^{T} \mid w_{2}\right]^{T} \in \partial G_{i}^{+} \cap \partial G_{i} .}
\end{aligned}
$$

The set $\partial G_{i}$ can intersect $\partial G_{i}^{+}$at the half-planes $\Gamma_{i 1} A_{w} w_{1}=$ $-\left(\Gamma_{i} B_{w}\right)\left( \pm \eta-\zeta^{*}\right)$ or at $w_{2}=0$. Therefore, we solve separate optimization problems for each case and enforce $w_{1}^{T *} P w_{1}^{*} \geq 1$ in both. Noting that the portion of $\partial G_{i}^{+}$contained in $w_{2}=0$ is defined by $\left|\Gamma_{i 1} A_{w} w_{1}-\Gamma_{i} B_{w} \zeta^{*}\right| \leq\left|\Gamma_{i} B_{w}\right| \eta$, we see that the optimization problems to be solved are

$$
\begin{aligned}
\min _{w_{2} \neq 0} w_{1}^{T} P w_{1} & \quad \text { s.t. } \\
\Gamma_{i 1} A_{w} w_{1} & =-\left(\Gamma_{i} B_{w}\right)\left(\eta \operatorname{sign}\left(\mathcal{G}_{w} w_{2}\right)-\zeta^{*}\right) \\
\Gamma_{i 1} w_{1}+\Gamma_{i 2} w_{2} & =1
\end{aligned}
$$

and

$$
\begin{gathered}
\min w_{1}^{T} P w_{1} \quad \text { s.t. } \\
\Gamma_{i 1} w_{1}=1 \\
\left|\Gamma_{i 1} A_{w} w_{1}-\Gamma_{i} B_{w} \zeta^{*}\right| \leq\left|\Gamma_{i} B_{w}\right| \eta .
\end{gathered}
$$

Define

$$
\begin{aligned}
M & =\left[\frac{F_{1}}{F_{2}}\right] P^{-1}\left[F_{1}^{T} \mid F_{2}^{T}\right] \\
F_{1} & =\Gamma_{i 1} A_{w} \\
F_{2} & =\Gamma_{i 1} .
\end{aligned}
$$

It is straightforward to see that $M$ is a symmetric, positive-definite $2 \times 2$ matrix when $\Gamma_{i 1} \neq 0$ and $\Gamma_{i 1} A_{w}$ and $\Gamma_{i 1}$ are linearly independent. The previous optimization problems have closed-form solutions, divided into several cases according to whether $M$ is singular or not and depending on certain condi-

\begin{tabular}{|c|c|c|}
\hline Case & Subcase & Condition \\
\hline \multirow[t]{7}{*}{$\Gamma_{i 1} A_{w} \nVdash \Gamma_{i 1}$} & $\begin{array}{l}\mathcal{G}_{w} w_{2, \text { crit }}^{+}>0, w_{2, \text { crit }}^{+} \in\left(\underline{w}_{2}, \bar{w}_{2}\right) \\
\text { and } \Gamma_{i 2} \neq 0\end{array}$ & $\mathrm{C} 1$ \\
\hline & $\begin{array}{l}\left(\mathcal{G}_{w} w_{2, \text { crit }}^{+} \leq 0 \text { and }\right. \\
\left.w_{2, \text { crit }}^{+} \in\left(\underline{w}_{2}, \bar{w}_{2}\right)\right) \text { or } \Gamma_{i 2}=0\end{array}$ & $\mathrm{C} 3$ \\
\hline & $\begin{array}{l}\mathcal{G}_{w} w_{2, \text { crit }}^{-}<0, w_{2, \text { crit }}^{-} \in\left(\underline{w}_{2}, \bar{w}_{2}\right) \\
\text { and } \Gamma_{i 2} \neq 0\end{array}$ & $\mathrm{C} 2$ \\
\hline & $\begin{array}{l}\left(\mathcal{G}_{w} w_{2, c r i t}^{-} \geq 0 \text { and }\right. \\
\left.w_{2, \text { crit }}^{-} \in\left(\underline{w}_{2}, \bar{w}_{2}\right)\right) \text { or } \Gamma_{i 2}=0\end{array}$ & $\mathrm{C} 4$ \\
\hline & $\left|\Gamma_{i 1} A_{w} \hat{w}_{1}-\Gamma_{i} B_{w} \zeta^{*}\right| \leq\left|\Gamma_{i} B_{w}\right| \eta$ & C7 \\
\hline & $\left|\Gamma_{i 1} A_{w} \hat{w}_{1}-\Gamma_{i} B_{w} \zeta^{*}\right|>\left|\Gamma_{i} B_{w}\right| \eta$ & $\begin{array}{l}\mathrm{C} 8 \\
\text { and C9 }\end{array}$ \\
\hline & otherwise & $\begin{array}{l}\text { constraint } \\
\text { satisfied }\end{array}$ \\
\hline \multirow[t]{5}{*}{$\Gamma_{i 1} A_{w}=\alpha \Gamma_{i 1}$} & $\begin{array}{l}\mathcal{G}_{w} \tilde{w}_{2}^{+}>0, \tilde{w}_{2}^{+} \in\left(\underline{w}_{2}, \bar{w}_{2}\right) \\
\text { and } \Gamma_{i 2} \neq 0\end{array}$ & $\mathrm{C} 5$ \\
\hline & $\begin{array}{l}\mathcal{G}_{w} \tilde{w}_{2}^{-}<0, \tilde{w}_{2}^{-} \in\left(\underline{w}_{2}, \bar{w}_{2}\right) \\
\text { and } \Gamma_{i 2} \neq 0\end{array}$ & C6 \\
\hline & $\Gamma_{i 2}=0$ and $\eta=\left|\zeta^{*}-\alpha /\left(\Gamma_{i} B_{w}\right)\right|$ & $\mathrm{C} 7$ \\
\hline & $\left|\alpha-\Gamma_{i} B_{w} \zeta^{*}\right| \leq\left|\Gamma_{i} B_{w}\right| \eta$ & $\mathrm{C} 7$ \\
\hline & otherwise & $\begin{array}{l}\text { constraint } \\
\text { satisfied }\end{array}$ \\
\hline
\end{tabular}
tions on the parameters. Readers are referred to [16] for details. Verification of Corollary 1 is achieved through the decision procedure shown in Tables I and II. The tables are used with the following variables:

$$
\begin{aligned}
w_{2, \text { crit }}^{+} & =\frac{\bar{M}_{22}-\bar{M}_{12} \Gamma_{i} B_{w}\left(\eta-\zeta^{*}\right)}{\Gamma_{i 2} \bar{M}_{22}} \\
w_{2, \text { crit }}^{-} & =\frac{\bar{M}_{22}-\bar{M}_{12} \Gamma_{i} B_{w}\left(-\eta-\zeta^{*}\right)}{\Gamma_{i 2} \bar{M}_{22}} \\
\tilde{w}_{2}^{+} & =\frac{1}{\Gamma_{i 2}}\left(1+\frac{\Gamma_{i} B_{w}\left(\eta-\zeta^{*}\right)}{\alpha}\right) \\
\tilde{w}_{2}^{-} & =\frac{1}{\Gamma_{i 2}}\left(1+\frac{\Gamma_{i} B_{w}\left(-\eta-\zeta^{*}\right)}{\alpha}\right) \\
\hat{w}_{1} & =\frac{P^{-1} \Gamma_{i 1}^{T}}{\Gamma_{i 1} P^{-1} \Gamma_{i 1}^{T}} \\
v_{1} & =\left[-\Gamma_{i} B_{w}\left(\eta-\zeta^{*}\right) 1\right] \\
v_{2} & =\left[\Gamma_{i} B_{w}\left(\eta+\zeta^{*}\right) 1\right] \\
v_{3} & =\left[\left|\Gamma_{i} B_{w}\right|(\eta-\bar{\zeta}) 1\right] \\
v_{4} & =\left[-\left|\Gamma_{i} B_{w}\right|(\eta+\bar{\zeta}) 1\right]
\end{aligned}
$$

TABLE I

CONDITION TABLE

\begin{tabular}{||l|l||}
\hline Label & Condition \\
\hline C1 & $\left(\eta-\zeta^{*}\right)^{2}>\frac{M_{11}}{\left(\Gamma_{i} B_{w}\right)^{2}}$ \\
\hline C2 & $\left(\eta+\zeta^{*}\right)^{2}>\frac{M_{11}}{\left(\Gamma_{i} B_{w}\right)^{2}}$ \\
\hline C3 & $v_{1} M^{-1} v_{1}^{T}>1$ \\
\hline C4 & $v_{2} M^{-1} v_{2}^{T}>1$ \\
\hline C5 & $\left(\frac{\Gamma_{i} B_{w}\left(\eta-\zeta^{*}\right)}{\alpha}\right)^{2}>M_{22}$ \\
\hline C6 & $\left(\frac{\Gamma_{i} B_{w}\left(\eta+\zeta^{*}\right)}{\alpha}\right)^{2}>M_{22}$ \\
\hline C7 & $\Gamma_{i 1} P^{-1} \Gamma_{i 1}^{T}<1$ \\
\hline C8 & $v_{3} M^{-1} v_{3}^{T}>1$ \\
\hline C9 & $v_{4} M^{-1} v_{4}^{T}>1$ \\
\hline
\end{tabular}

TABLE II

DECISION TABLE

where $\bar{M}_{i j}$ are the entries of $M^{-1}$ and $M_{i j}$ are those of $M$. It is straightforward to show that if $\Gamma_{i 1} A_{w}=0$ then the $i$ th constraint is automatically satisfied.

\section{E. Design Philosophy and Control Constraints}

The method presented here does not specifically address regulation performance. The results are useful to guarantee that the specified state constraints will not be violated as the state proceeds to the origin under the SMC law. All this is done in the presence of a disturbance. The following steps summarize the basic method.

1) Select the coefficients of the sliding hyperplane $G$ based on dynamic specifications for the sliding mode.

2) Obtain the $w$-coordinate description of the closed-loop system (8) using matrix $J=T K$.

3) Use either the LMI method or the direct critical switching gain method to find an RPI cylinder and the maximum sum of switching gain $\eta$ and disturbance bound $\bar{\zeta}$. Alternatively, if $\eta$ has been selected based on other considerations (such as reaching time), evaluate the maximum allowable disturbance to preserve RPI. 
4) Use Tables I and II to qualify each constraint. If all constraints are satisfied, their intersection with the RPI cylinder is the safe operating set.

5) If a larger operating set is sought, a larger RPI cylinder may be sought in step 3 (see the following).

Several refinements can be incorporated to aid the solution of LMI (14). For instance, one may rule out large ellipsoids, e.g., those whose interior contains the constraint set. This is accomplished by enforcing $P>k I$, where $k$ is a suitable positive scalar to be determined from constraint geometry. The volume of the ellipsoid can be maximized under $P>k I$ and the LMI constraint. The problem formulation in this case becomes

$$
\begin{aligned}
& \min \operatorname{trace}(P) \quad \text { s.t. }
\end{aligned}
$$

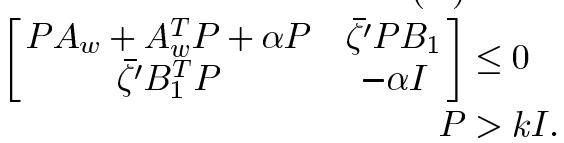

Volume optimization is included in the numerical example of Section VII. Control constraints are easily incorporated in the design. In fact, the control law of (4) can be expressed in $x$-coordinates as

$$
u=-\mathcal{G} A x-\eta \operatorname{sign}(s) .
$$

Thus, it is straightforward to show, using the triangle inequality, that a control constraint of the form $|u| \leq \bar{u}$ can be accommodated by introducing the additional state constraints $\Gamma_{u} w \leq 1$ and $-\Gamma_{u} w \leq 1$, where

$$
\Gamma_{u}=\frac{\mathcal{G} A J}{\bar{u}-\eta} .
$$

\section{REMOVAL OF CHATTERING BY BOUNDARY LAYER}

Total insensitivity to matched disturbance during the sliding motion is obtained at the cost of rapid control switching across the sliding manifold. Such chattering may not be allowed in a practical realization of the SMC controller due to actuator wear and excitation of high-frequency unmodeled dynamics of the plant. The chattering problem has been addressed in a variety of ways [4], [19], [20]. In this paper, we choose the commonly used finite-slope approximation of the switching law. This allows us to exploit the inherent robust positive invariance of the boundary layer and introduce minor changes to the results derived for the switching case. That is, we replace the signum function in (4) with the saturation function

$$
\operatorname{sat}\left(\frac{s}{\phi}\right)=\left\{\begin{array}{lr}
\frac{s}{\phi}, & \text { when }|s| \leq \phi \\
\operatorname{sign}(s), & \text { when }|s|>\phi
\end{array}\right.
$$

where $\phi$ represents the boundary layer thickness. The closedloop dynamics are now described by (8), substituting $\operatorname{sign}(s)$ by $\operatorname{sat}(s / \phi)$. We will refer to the new dynamics as $(8)_{\phi}$. Although it is possible and sometimes useful to let $\phi$ vary with time to better manage the tradeoff between control bandwidth and accuracy, we will assume that $\phi$ is a constant positive quantity. The set

$$
\begin{aligned}
\mathcal{B}_{\phi} & \triangleq\left\{w \in \mathbb{R}^{n}:|s(w)| \leq \phi\right\} \\
& =\left\{w \in \mathbb{R}^{n}:\left|w_{2}\right| \leq\left|B_{2}\right| \phi\right\}
\end{aligned}
$$

is called the boundary layer.

\section{A. Closed-Loop Dynamics in $\mathcal{B}_{\phi}$}

When $|s(w)| \leq \phi$, the dynamics in $w$-coordinates become

$$
\begin{aligned}
& \dot{w}_{1}=A_{w} w_{1}+B_{1}\left(-\frac{\eta}{\phi B_{2}} w_{2}-\zeta(t)\right) \\
& \dot{w}_{2}=-\frac{\eta}{\phi} w_{2}-B_{2} \zeta(t) .
\end{aligned}
$$

The dynamics of $w_{1}$ now depend on $w_{2}$ through a linear function. In the switching case of (8), full decoupling was achieved at either side of $w_{2}=0$. Also, the dynamics of $w_{2}$ is now linear. It can be easily shown that, when $\eta>\bar{\zeta}$ is properly selected, the interval $\left[-B_{2} \phi, B_{2} \phi\right]$ is RPI with respect to the dynamics of $w_{2}$ in (21). Also, $\mathcal{B}_{\phi}$ is attractive, that is, all trajectories of $8_{\phi}$ enter $\mathcal{B}_{\phi}$ in finite time and remain there for all future times despite the presence of the uncertainty. We summarize the results in the following.

Lemma 6: Let $\eta>\bar{\zeta}$. Then, for any initial condition $w(0) \in$ $\mathbb{R}^{n}, \exists t_{r}>0$ such that the trajectory $w(t)$ of $8_{\phi}$ satisfies $w(t) \in$ $\mathcal{B}_{\phi}$ for all $t \geq t_{r}$.

Note that only the interval $\left[-\left|B_{2}\right| \phi,\left|B_{2}\right| \phi\right]$ (and not an arbitrary subinterval) is guaranteed to be RPI. Lemma 6 plays the role of Lemma 2 in our earlier derivations. Similarly, the following result analogous to Theorem 5 applies.

Theorem 6: Let $\mathcal{S}_{o} \subset \mathbb{R}^{n-1}$ be a compact and convex set containing the origin. Suppose $\mathcal{S}_{o}$ is RPI for the system $\dot{w}_{1}=$ $A_{w} w_{1}+B_{1} \zeta^{\prime}(t)$, where $A_{w}$ is Hurwitz and $\left|\zeta^{\prime}(t)\right| \leq \bar{\zeta}+\left(\eta / B_{2}\right)$ for all $t \geq 0$. Then, all cylinders $\mathcal{H}_{\mathcal{S}_{o}}[a, b]$ such that $[a, b] \subset$ $\left[-\left|B_{2}\right| \phi,\left|B_{2}\right| \phi\right]$ are RPI for the closed-loop dynamics of $8_{\phi}$.

In fact, $w_{2}(t)$ in (20) can be seen as a bounded perturbation for the dynamics of $w_{1}$, that is, $\left|w_{2}(t)\right| \leq \phi$ for $t>t_{r}$, due to invariance. Theorem 6 introduces little modification to the critical switching gain or LMI methods of Lemmas 5 and 4. Given a $P$ matrix corresponding to the cross section of a cylinder designed for the switching case, invariance of the cylinder within the boundary layer is checked by substituting $\eta+\bar{\zeta}$ by $\bar{\zeta}+\left(\eta /\left|B_{2}\right|\right)$ in the definition of $\zeta^{\prime}$. The LMI must hold for some value of $\alpha$ (not necessarily the same one used for the switching case) or, alternatively, the inequality of Lemma 4 must hold.

\section{B. Incorporation of State Constraints}

Define the following vector field associated with the dynamics (20) and (21):

$$
\begin{gathered}
f_{\phi}(w, \zeta) \triangleq\left[\left(A_{w} w_{1}+B_{1}\left(-\frac{\eta}{\phi B_{2}} w_{2}-\zeta\right)\right)^{T} \mid\right. \\
\left.-\frac{\eta}{\phi} w_{2}-B_{2} \zeta(t)\right]^{T}
\end{gathered}
$$

and introduce the sets

$$
\begin{aligned}
& G_{\phi i}^{+}=\left\{w \in \mathcal{B}_{\phi}: \Gamma_{i} f_{\phi}(w, \zeta)>0 \text { for some } \zeta \in \mathcal{Z}\right\} \\
& G_{\phi i}^{-}=\left\{w \in \mathcal{B}_{\phi}: \Gamma_{i} f_{\phi}(w, \zeta)<0 \text { for all } \zeta \in \mathcal{Z}\right\} .
\end{aligned}
$$

The counterpart of Corollary 1 of Section IV can be stated as follows. 


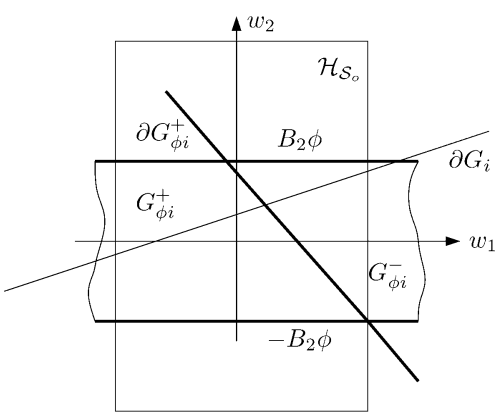

Fig. 2. RPI state-constrained cylinder.

Corollary 2: Let the boundary layer $\mathcal{B}_{\phi}$ be RPI for the dynamics of (20) and (21). Then, $\mathcal{S}_{\phi}=\mathcal{H}_{\mathcal{S}_{o}}\left[-\left|B_{2}\right| \phi,\left|B_{2}\right| \phi\right] \cap G$ is RPI for the same dynamics if $\mathcal{H}_{\mathcal{S}_{o}}\left[-\left|B_{2}\right| \phi,\left|B_{2}\right| \phi\right] \cap \partial G_{i} \cap$ $\partial G_{\phi i}^{+}=\varnothing$ for all $i=1,2 \ldots m$.

Corollary 2 can be proved with the aid of the following results.

Lemma 7: Let the boundary layer $\mathcal{B}_{\phi}$ be RPI for the dynamics (20) and (21). Let a linear state constraint $G_{i}$ be such that $\partial G_{i} \cap$ $\mathcal{H}_{\mathcal{S}_{o}}\left[-\left|B_{2}\right| \phi,\left|B_{2}\right| \phi\right] \neq \varnothing$. Then, $\exists w \in \mathcal{H}_{\mathcal{S}_{o}}\left[-\left|B_{2}\right| \phi,\left|B_{2}\right| \phi\right] \cap$ $\partial G_{i}$ such that $\Gamma_{i} f_{\phi}(w, \zeta)<0 \forall \zeta \in \mathcal{Z}$.

Lemma 7 is proved in a manner similar to Lemma 3.

Proposition 2: Suppose $\Gamma_{i}=\left[\Gamma_{i 1} \mid \Gamma_{i 2}\right]$, where $\Gamma_{i 1} A_{w} \neq 0$. Then, the collection of subsets $\left\{G_{\phi i}^{+}, G_{\phi i}^{-}, \partial G_{\phi i}^{+}\right\}$corresponding to $f_{\phi}(w, \zeta)$ of $(22)$ is a partition of $\mathcal{B}_{\phi}$. Furthermore

$$
\partial G_{\phi i}^{+}=\left\{w \in \mathcal{B}_{\phi}: \Gamma_{i} A_{w} w_{1}=\frac{\eta \Gamma_{i} B_{w}}{B_{2} \phi} w_{2}-\left|\Gamma_{i} B_{w}\right| \bar{\zeta}\right\} .
$$

The proof of Proposition 2 is straightforward. Fig. 2 illustrates the partition. The constraint boundary shown intersects $\partial G_{\phi i}^{+}$within $\mathcal{B}_{\phi}$, indicating constraint violation in the boundary layer. Corollary 2 is proved in the same way as Corollary 1, using Theorem 4 and taking $M=\mathcal{H}_{\mathcal{S}_{o}}\left[-\left|B_{2}\right| \phi,\left|B_{2}\right| \phi\right]$ and $\mathcal{S}_{\phi}=G \cap M$. The result guarantees that the constraints will not be violated for system trajectories contained in the boundary layer. We must, however, establish robust invariance of the whole constrained cylinder, taking into account the change in dynamics introduced by the saturation function. We assume that a design has been completed assuming the original switching dynamics of (8) and that, in addition, Corollary 2 is satisfied. The following result formalizes the validity of the approach.

Theorem 7: Let $H \in \mathcal{H}_{\mathcal{S}_{o}}$ and suppose $G \cap H$ is RPI for the dynamics of (8). If $G \cap \mathcal{H}_{\mathcal{S}_{o}}\left[-\left|B_{2}\right| \phi,\left|B_{2}\right| \phi\right]$ is RPI for dynamics (20) and (21), then $G \cap H$ is RPI for the dynamics of $8_{\phi}$.

The previous theorem is directly proved by examining an arbitrary trajectory starting in $\mathcal{H}_{\mathcal{S}_{o}} \cap G$ and using the hypotheses and attractiveness of the boundary layer. Standard causality and time-invariance arguments allow the concatenation of the subtrajectories corresponding to motion inside and outside the boundary layer.

\section{Ellipsoidal Cross Sections}

For cylinders with ellipsoidal cross section, the results of Corollary 2 can be reduced to computational steps. Assuming that the boundary layer has been rendered RPI with respect to the dynamics (20) and (21), design conditions can be obtained by finding the point $w^{*}$ of $\partial G_{\phi i}^{+} \cap \partial G_{i}$ (if any) for which $w_{1}^{T} P w_{1}$ is minimum and enforce $w_{1}^{T *} P w_{1}^{*}>1$. In other words, we solve the optimization problem

$$
\begin{aligned}
& \min _{w_{2} \in\left[-B_{2} \phi, B_{2} \phi\right]} w_{1}^{T} P w_{1} \quad \text { s.t. } \\
& {\left[w_{1}^{T} \mid w_{2}\right]^{T} \in \partial G_{\phi i}^{+} \cap \partial G_{i} .}
\end{aligned}
$$

As in the switching case, the problem has a straightforward closed-form solution which leads to a few inequalities to be checked. Based on Theorem 7, it is evident that these inequalities must be checked in addition to those of Tables I, II. Of course, the RPI condition in the boundary layer must hold. The additional inequalities contain $\phi$ as a parameter. The simplification of the whole set of inequalities is still a work in progress. Some simplification is expected if constraint symmetry is assumed.

\section{NUMERICAL EXAMPLE}

Consider the following controllable pair:

$$
A=\left[\begin{array}{ccc}
-1 & 0 & 0 \\
1 & -2 & -1 \\
0 & 1 & 0
\end{array}\right] \quad B=\left[\begin{array}{l}
1 \\
0 \\
1
\end{array}\right]
$$

Consider that the constraints in $x$-coordinates are given by a parallelepiped containing the origin in its interior. The rows $\Gamma_{x}$ specify individual constraints

$$
\Gamma_{x}=\left[\begin{array}{ccc}
0.8 & 0.32 & -0.16 \\
-1 & -0.4 & 0.2 \\
0 & 0.96 & 0.32 \\
0 & -1.2 & -0.4 \\
0 & 0.4 & 0.8 \\
0 & -0.5 & -1
\end{array}\right]
$$

Suppose that the disturbance is given by $\zeta(t)=0.2 \sin (10 t)$. An appropriate set of transformation matrices is given by

$$
\begin{aligned}
T & =\left[\begin{array}{ccc}
1 & -1 & 1 \\
0 & 1 & 0 \\
0 & -1 & 1
\end{array}\right] \quad K=\left[\begin{array}{ccc}
1 & 0 & -1 \\
0 & 1 & -0.5 \\
0 & 0.5 & 1
\end{array}\right] \\
J & =T K .
\end{aligned}
$$

The transformed constraints are obtained as the rows of $\Gamma_{x} J$. Note that the constraints are not symmetric in either $x$ - or $w$-coordinates. Using the transformation $x=T K w=J w$, matrix $A_{11}$ has two negative eigenvalues. Choosing to place the poles of $A_{w}$ at $[-1.5 \pm 0.5 i]$ results in $\mathcal{G}_{1}=[0-0.5]$, which corresponds to $\mathcal{G}=\left[\begin{array}{lll}0 & 0.5 & 1\end{array}\right]$ in the original $x$-coordinates. The range for $\alpha$ is $(0,3)$. Choosing an arbitrary fixed $\alpha=2.1$, we solve the volume optimization problem using $k=0.5$ and $\eta+\bar{\zeta}=1$. The solution is a matrix $P$ with $[0.8289,0.6584]$ being the diagonal and -0.2283 the off-diagonal. Choosing $\eta=0.8$ satisfies the decision procedure for constraint qualification and thus the intersection of the cylinder and state constraints in $w$-coordinates is RPI. Of course, the intersection of the transformed cylinder 


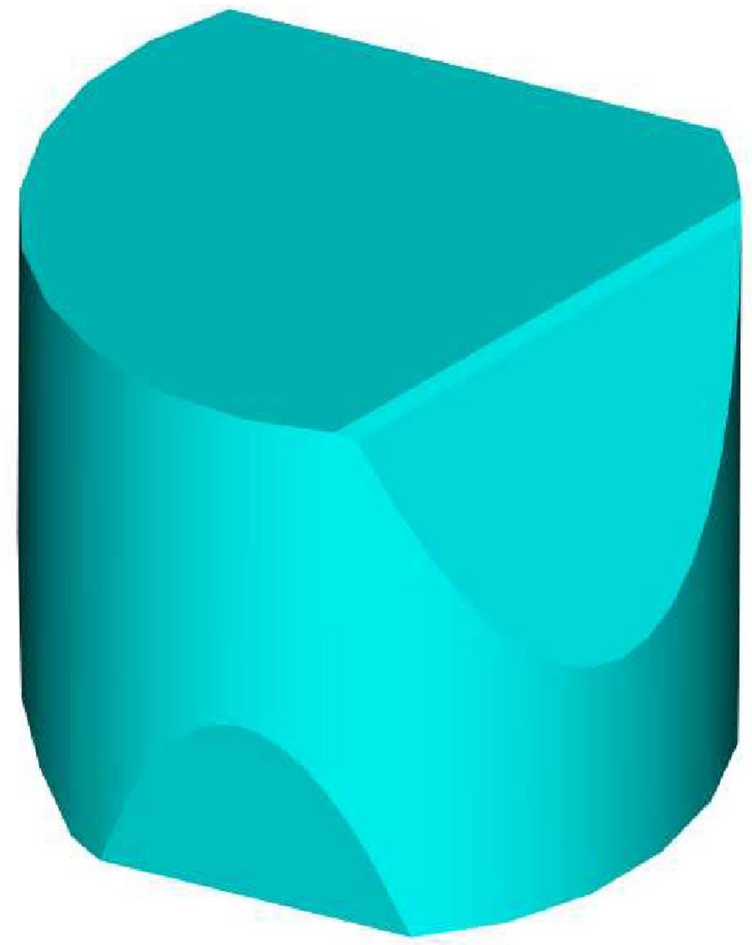

Fig. 3. RPI state-constrained cylinder.

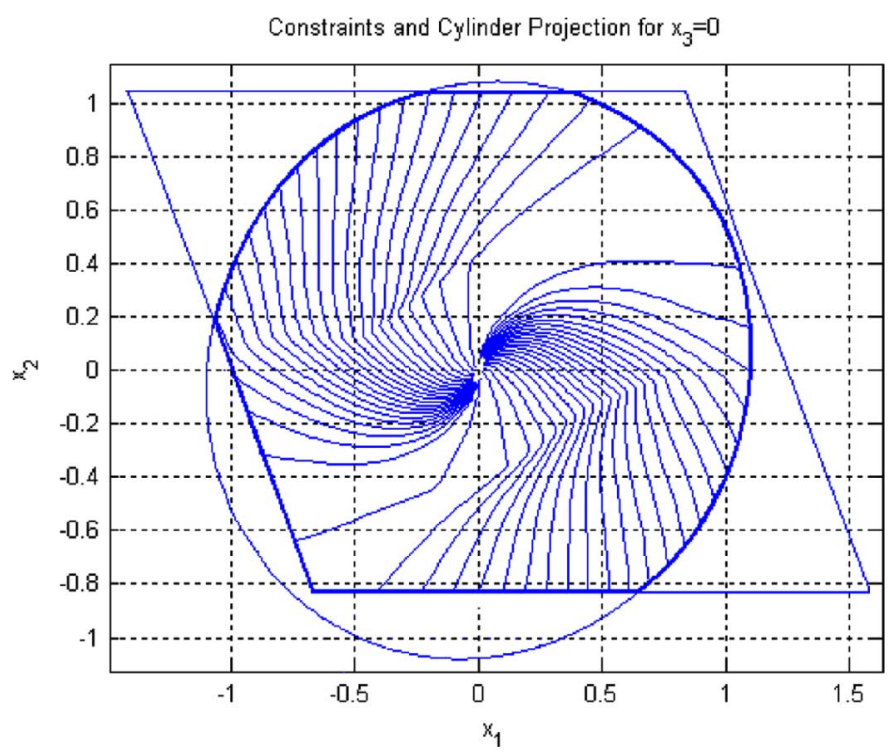

Fig. 4. Projected trajectories and constraints.

and constraints in $x$-coordinates is also RPI. Using the alternative method with the same $P$ matrix, we obtain $\beta=2.3324$ and

$$
\eta_{\text {crit }}+\bar{\zeta}=\frac{\beta}{2 \sqrt{B_{1}^{T} P_{w} B_{1}}}=1.6667
$$

Then, we may choose, for instance, $\eta=1.4$ for ellipsoidal invariance alone, but there is no guarantee that this choice of gain will satisfy the decision procedure for all constraints. In fact, the second constraint would be violated with $\eta=1.4$. Fig. 3 sketches the shape of constrained cylinder in $w$-coordinates. Fig. 4 sketches, in $x$-coordinates, the trajectories projected onto the $x_{3}=0$ plane and the constraints and transformed cylinder section at $x_{3}=0$. If the saturation function is used with $\phi=1$, it can be verified that the LMI condition of (14) still holds for $\zeta^{\prime}=\bar{\zeta}+\left(\eta / B_{2}\right)=1.2$, using $\alpha=1.9$. This guarantees that the cylinder is still RPI for the boundary layer dynamics. Note that the RPI condition alone is independent of $\phi$. It can be verified that a value of $\phi=1$, for instance, satisfies the set of additional inequalities required for constraint satisfaction inside the boundary layer.

\section{EXTENSIONS}

Several extensions to the general cylinder approach are possible. In this section, we focus in a restricted kind of nonlinear plants. Certain kinds of nonlinear system under SMC with linear manifolds have reaching dynamics similar to that of linear systems. Single-input nonlinear systems in integrator chain form are a direct example. Consider the single-input system

$$
\begin{aligned}
\dot{x}_{1}= & x_{2} \\
& : \\
\dot{x}_{n-1}= & x_{n} \\
\dot{x}_{n}= & f(x)+g(x) u
\end{aligned}
$$

where $g(x) \neq 0$ in a convex region of $\mathbb{R}^{n}$ containing the origin. As in the linear case, let the sliding function be defined by $s=$ $\mathcal{G} x$. Suppose the control input $u$ is such that

$$
\dot{s}=-\eta \operatorname{sign}(s)
$$

with $\eta>0$. Assume without loss of generality (w.l.o.g.) that the $n$th component of $\mathcal{G}$ is 1 . Then, the closed-loop dynamics can be expressed as

$$
\begin{aligned}
{\left[\begin{array}{c}
\dot{x}_{1} \\
\vdots \\
\dot{x}_{n-1} \\
\dot{x}_{n}
\end{array}\right]=} & {\left[\begin{array}{cccc}
0 & 1 & \cdots & 0 \\
\vdots & \cdots & & \vdots \\
0 & 0 & \cdots & 1 \\
0 & -\mathcal{G}_{1} & \cdots & -\mathcal{G}_{n-1}
\end{array}\right]\left[\begin{array}{c}
x_{1} \\
\vdots \\
x_{n-1} \\
x_{n}
\end{array}\right] } \\
& -\left[\begin{array}{c}
0 \\
\vdots \\
0 \\
1
\end{array}\right] \eta \operatorname{sign}(s) \\
s= & \mathcal{G} x .
\end{aligned}
$$

When sliding occurs, $\dot{x}_{n}=-\left[\mathcal{G}_{1} \mathcal{G}_{2} \cdots \mathcal{G}_{n-1}\right] \cdot\left[x_{2} \cdots x_{n}\right]^{T}$ and the last state equation in (25) is redundant with the sliding condition $\dot{s}=0$. The reduced dynamics is described by

$$
\left[\begin{array}{c}
\dot{x}_{1} \\
\vdots \\
\dot{x}_{n-2} \\
\dot{x}_{n-1}
\end{array}\right]=\left[\begin{array}{cccc}
0 & 1 & \cdots & 0 \\
\vdots & \cdots & & \vdots \\
0 & 0 & \cdots & 1 \\
-\mathcal{G}_{1} & -\mathcal{G}_{2} & \cdots & -\mathcal{G}_{n-1}
\end{array}\right]\left[\begin{array}{c}
x_{1} \\
\vdots \\
x_{n-2} \\
x_{n-1}
\end{array}\right] .
$$

Define the constant matrix in (26) as $A_{w}$. This matrix has the standard controllability form, and it is clear how to choose the first $n-1$ coefficients of $\mathcal{G}$ to achieve a stable sliding mode. 
In the following, it will be assumed that $A_{w}$ is Hurwitz. As in the linear case, we seek a coordinate transformation which reveals the singular structure of the constant matrix in (25). The existence of such transformation is ascertained in the following.

Lemma 8: There exists a coordinate transformation $x=J w$ with $J$ nonsingular in which the closed-loop dynamics (25) is expressed as

$$
\left\{\begin{array}{l}
{\left[\begin{array}{l}
\dot{w}_{1} \\
\dot{w}_{2}
\end{array}\right]=\left[\begin{array}{l|l}
A_{w} & 0 \\
\hline 0 & 0
\end{array}\right]\left[\frac{w_{1}}{w_{2}}\right]+\left[\frac{B_{1}}{-1}\right] \eta \operatorname{sign}(s)} \\
s=w_{n}
\end{array}\right.
$$

where $B_{1}=[0 \ldots 1]^{T}$.

One possible form of $J$ that verifies Lemma 8 is given by

$$
J^{-1}=\left[\begin{array}{cccc}
0 & 1 & \cdots & 0 \\
\vdots & \cdots & \vdots & \\
0 & 0 & \vdots & 1 \\
\mathcal{G}_{1} & \mathcal{G}_{2} & \cdots & 1
\end{array}\right] .
$$

Once the system is written in $w$-coordinates, it is straightforward to find invariant cylinders with the methods of previous sections. In addition to the previous nonlinear extension, it is observed that certain minimum-phase, nonlinear multiple-input-multiple-output (MIMO) systems under tracking control via sliding modes result in decoupled tracking error dynamics which essentially reduce to the forms contemplated in this paper and can be, therefore, treated with the methods described here. Multivariable extensions are certainly possible.

\section{CONClusion}

General results are given concerning robust positive invariance of the intersection of a primary invariant set with a convex state constraint set. RPI cylinders are introduced as a tool to design sliding-mode controllers for constrained linear systems. In the case of ellipsoidal cross sections, we provide conditions under which the cylinder is RPI in terms of a linear matrix inequality. Further, a decision procedure is described that qualifies each constraint so that the constrained cylinder is itself RPI. The conditions reduce to a few inequalities on the switching gain. The number of inequalities to check depends on the number of constraints, but not on system order. Removal of chattering is addressed by introducing a finite-slope approximation to the switching function. The treatment of this case fits in the cylinder framework. Additional conditions are given so that an already designed cylinder for the switching case can still be used. Certain nonlinear plants under SMC with linear manifold also fit in the cylinder framework. Other extensions to explore include robust output tracking, multiple-input systems and unmatched disturbance.

\section{APPENDIX I \\ CONSTRUCTION OF $T$}

One possible way to select $T$ is presented here. First, find a transformation $T_{1}$ to put $(A, B)$ in regular form using, for instance, the QR decomposition method shown in [14]. In this method, the nonzero component of $T_{1}^{-1} B$ is not guaranteed to be 1 , as required in this paper. A new $T_{1}$, however, can be easily obtained by scaling. Denote the $(1,1)$ block component of $T_{1}^{-1} A T_{1}$ by $a_{11}$. If $a_{11}$ is invertible, the required transformation has been found. If not, a second transformation $S$ is applied to the regular form found previously. The regular form matrices have the form

$$
\begin{aligned}
T_{1}^{-1} A T_{1} & =\left[\begin{array}{l|l}
a_{11} & a_{12} \\
\hline a_{21} & a_{22}
\end{array}\right] \\
T_{1}^{-1} B & =\left[\begin{array}{c}
0 \\
b_{2}
\end{array}\right] .
\end{aligned}
$$

Note that the regular form has the properties of $\left(a_{11}, a_{12}\right)$ being controllable and $b_{2} \neq 0$ [14]. Partition $S^{-1}$ into four compatible blocks named $S_{i j}, i, j=1,2$. Then, we must have

$$
\begin{aligned}
& S_{12} b_{2}=0 \\
& S_{22} b_{2}=1 .
\end{aligned}
$$

Set $S_{11}$ to any invertible $n-1 \times n-1$ matrix, set $S_{12}=0$, and let

$$
S_{22}=\frac{1}{b_{2}}
$$

Selection of $S_{21}$ can be done so that the $(1,1)$ block of $S^{-1}\left(T_{1}^{1} A T_{1}\right) S$ has desired eigenvalues, specifically, to make it nonsingular. Let $T=T_{1} S$ and denote the four block components of $T^{-1} A T$ by $A_{i j}, i, j=1,2$. Using the block matrix inversion formulas found, for instance, in [21, App.], it is possible to show that

$$
\begin{aligned}
& A_{11}=S_{11}\left(a_{11}-a_{12} b_{2} S_{21}\right) S_{11}^{-1} \\
& A_{12}=S_{11} a_{12} b_{2} .
\end{aligned}
$$

Since $\left(a_{11}, a_{12}\right)$ is controllable and $b_{2} \neq 0$, it follows directly that $\left(a_{11}, a_{12} b_{2}\right)$ is also controllable. Thus, $S_{21}$ can be used to assign nonzero eigenvalues to $A_{11}$. Therefore, $T=T_{1} S$ is the sought transformation. Finally, we show that $\left(A_{11}, A_{12}\right)$ is controllable. In fact, $\left(A_{11}, A_{12}\right)$ has the same controllability as $\left(a_{11}-a_{12} b_{2} S_{21}, a_{12} b_{2}\right)$, since the two pairs are related by similarity transformation. Given that the system associated with $\left(a_{11}, a_{12}\right)$ is controllable, the feedback transformation $u \mapsto$ $b_{2}\left(u-S_{21} x\right)$ shows that $\left(a_{11}-a_{12} b_{2} S_{21}, a_{12} b_{2}\right)$ is also controllable.

\section{APPENDIX II PROOF OF THEOREM 4}

Sufficiency: In view of Theorem 2, we wish to show that $f(w, \zeta) \in \mathcal{C}_{\mathcal{S}}(w) \forall w \in \partial \mathcal{S}, \forall \zeta \in \mathcal{Z}$. It is possible to show [16], under the convexity and closure assumptions, that one can decompose the boundary of $\mathcal{S}$ as

$$
\partial \mathcal{S}=(M \cap \partial G) \cup(\partial M \cap G) .
$$

First, we show that $f(w, \zeta) \in \mathcal{C}_{\mathcal{S}}(w) \forall w \in(M \cap \partial G)$. By hypothesis, $\Gamma_{i} f(w, \zeta) \leq 0 \forall w \in \mathcal{S} \cap \partial G_{i}, \forall \zeta \in \mathcal{Z}, i=1,2 \cdots m$. Noting that $\bigcup\left(\mathcal{S} \cap \bar{\partial} G_{i}\right)=M \cap \partial G$, we have that $f(w, \zeta) \in$ $\mathcal{C}_{G}(w) \forall w \in(M \cap \partial G)$. Now, since $\partial G \cap M \subset M$, we have that, if $w \in \operatorname{int}(M)$ then $\mathcal{C}_{M}(w)=\mathbb{R}^{n}$, thus $f(w, \zeta) \in \mathcal{C}_{M}(w)$, whereas if $w \in \partial M$ then $f(w, \zeta) \in \mathcal{C}_{M}(w)$ due to $M$ being 
RPI. Therefore, $f(w, \zeta) \in \mathcal{C}_{M}(w) \cap \mathcal{C}_{G}(w) \forall w \in \partial G \cap M$. By assumption, $0 \in \operatorname{int}(M-G)$. Then, by Property $1, \mathcal{C}_{M}(w) \cap \mathcal{C}_{G}(w)=\mathcal{C}_{M \cap G}(w)=\mathcal{C}_{\mathcal{S}}(w)$. Thus, $f(w, \zeta) \in \mathcal{C}_{\mathcal{S}}(w) \forall w \in(M \cap \partial G)$. Now, we show that $f(w, \zeta) \in \mathcal{C}_{\mathcal{S}}(w) \forall w \in(G \cap \partial M)$. $M$ is RPI by assumption, therefore, $f(w, \zeta) \in \mathcal{C}_{M}(w) \forall w \in \partial M$, in particular, $\forall w \in \partial M \cap G$. Since $\partial M \cap G \subset G$, we have that, if $w \in \operatorname{int}(G)$ then $\mathcal{C}_{G}=\mathbb{R}^{n}$, thus $f(w, \zeta) \in \mathcal{C}_{G}(w)$, whereas if $w \in \partial G_{i}$ for some $i$, then $w \in \partial M \cap \partial G_{i} \subset \partial G_{i} \cap M$, since $M$ is closed. By hypothesis, it follows, as before, that $f(w, \zeta) \in \mathcal{C}_{G}(w)$. Thus, $f(w, \zeta) \in \mathcal{C}_{M}(w) \cap \mathcal{C}_{G}(w)=\mathcal{C}_{\mathcal{S}}(w) \forall w \in \partial M \cap G$. We conclude that $f(w, \zeta) \in \mathcal{C}_{\mathcal{S}}(w) \forall w \in \partial \mathcal{S}, \forall \zeta \in \mathcal{Z}$, and that $\mathcal{S}$ is, therefore, RPI.

Necessity: Suppose $\mathcal{S}$ is RPI, and by contradiction, suppose $\exists w \in \mathcal{S} \cap \partial G_{i}, \exists \zeta \in \mathcal{Z}$ for some $i$ such that $\Gamma_{i} f(w, \zeta)>0$. Then, $f(w, \zeta) \notin \mathcal{C}_{G_{i}}(w) \cap \mathcal{C}_{M}(w)$; so $\exists w \in \mathcal{S} \cap \partial G, \exists \zeta \in \mathcal{Z}$ such that $f(w, \zeta) \notin \mathcal{C}_{G}(w) \cap \mathcal{C}_{M}(w)=\mathcal{C}_{\mathcal{S}}(w)$. Since $\mathcal{S} \cap \partial G \subset$ $\partial \mathcal{S}$, we have that $\exists w \in \partial \mathcal{S}, \exists \zeta \in \mathcal{Z}$ such that $f(w, \zeta) \notin \mathcal{C}_{\mathcal{S}}(w)$, contradicting that $\mathcal{S}$ is RPI.

\section{APPENDIX III}

\section{PRoOF OF LEMMA 3}

Partition $\Gamma_{i}$ as $\Gamma_{i}=\left[\Gamma_{i 1} \mid \Gamma_{i 2}\right]$. Let $\partial G_{i}=\left\{\left[w_{1}^{T} \mid w_{2}\right]^{T} \in\right.$ $\left.\mathbb{R}^{n}: \Gamma_{i 1} w_{1}+\Gamma_{i 2} w 2=1\right\}$. Suppose $\Gamma_{i 2} \neq 0$. Then, $\partial G_{i}$ can be parameterized as $\left\{\left[w_{1}^{T} \mid w_{2}\right]^{T}\right.$ : $\left.w_{2}=\left(1-\Gamma_{i 1} w_{1} / \Gamma_{i 2}\right), w_{1} \in \mathbb{R}^{n-1}\right\}$. Consider the set $V=\left\{w_{2} \in \mathbb{R}: w_{2}=\left(1-\Gamma_{i 1} w_{1} / \Gamma_{i 2}\right), w_{1} \in \mathcal{S}_{o}\right\}$. The functional $h: \mathbb{R}^{n-1} \rightarrow \mathbb{R}$ given by $h\left(w_{1}\right)=\left(-\Gamma_{i 1} / \Gamma_{i 2}\right) w_{1}$ is bounded and, therefore, continuous in $\mathbb{R}^{n-1}$. Then, $h\left(\mathcal{S}_{o}\right)$ is compact, since $\mathcal{S}_{o} \subset \mathbb{R}^{n-1}$ is compact [22]. Thus, $V$, the translation of $h\left(\mathcal{S}_{o}\right)$, is also closed and bounded, i.e., $V$ is a real closed interval possessing a minimum and a maximum. Note also that the maximum and minimum values of $V$ are achieved at the boundary of $\mathcal{S}_{0}$. Denote by $w_{2}^{+}$ and $w_{2}^{-}$the half-spaces of $\mathbb{R}^{n}$ where $w_{2}>0$ and $w_{2}<0$, respectively. Denote by $w_{2}^{o}$ the plane $w_{2}=0$. Consider, first, the case when $\partial G_{i} \cap \mathcal{H}_{\mathcal{S}_{o}}\left[\bar{w}_{2}, \bar{w}_{2}\right] \cap w_{2}^{-}=\emptyset$. Let $b=\min (V)>0$. Note that $\bar{w}_{2} \geq b>\underline{w}_{2}$. Then, $\mathcal{H}_{\mathcal{S}_{o}}\left[\underline{w}_{2}, b\right]$ is RPI as a member of the family $\overline{\mathcal{H}}_{\mathcal{S}_{o}}$. This implies, by Theorem 2 that $f(w, \zeta) \in \mathcal{C}_{\mathcal{H}_{\mathcal{S}_{o}}\left[\underline{w}_{2}, b\right]}(w) \forall w \in$ $\partial \mathcal{H}_{\mathcal{S}_{o}}\left[\underline{w}_{2}, b\right], \forall \zeta \in \mathcal{Z}$. Now, $\mathcal{H}_{\mathcal{S}_{o}}\left[\underline{w}_{2}, b\right] \subset G_{i} \cap \mathcal{H}_{\mathcal{S}_{o}}\left[\underline{w}_{2}, \bar{w}_{2}\right]$, thus $\mathcal{C}_{\mathcal{H}_{\mathcal{S}_{o}}\left[\underline{w}_{2}, b\right]}(w) \subset \mathcal{C}_{G \cap \mathcal{H}_{\mathcal{S}_{o}}\left[\underline{w}_{2}, \bar{w}_{2}\right]}(w)$, in particular, for a point $w_{b} \stackrel{\underline{w}_{2}}{=}\left[w_{1}^{T} \mid b\right] \in \partial \mathcal{H}_{\mathcal{S}_{o}}\left[\underline{w}_{2}, b\right]$, where $w_{1} \in \partial \mathcal{S}_{o}$; so $f\left(w_{b}, \zeta\right) \in \mathcal{C}_{G \cap \mathcal{H}_{\mathcal{S}_{o}}\left[w_{2}, \bar{w}_{2}\right]}\left(w_{b}\right)=\mathcal{C}_{G_{i}}\left(w_{b}\right) \cap \mathcal{C}_{\mathcal{H}_{\mathcal{S}_{o}}\left[w_{2}, \bar{w}_{2}\right]}\left(w_{b}\right)$. Therefore, $\Gamma_{i} f\left(w_{b}, \zeta\right) \leq 0$, with $w_{b} \in \partial G_{i} \cap \partial \mathcal{H}_{\mathcal{S}_{o}}\left[\underline{w}_{2}, \bar{w}_{2}\right] \subset$ $\partial G_{i} \cap \mathcal{H}_{\mathcal{S}_{o}}\left[\underline{w}_{2}, \bar{w}_{2}\right]$. To prove the strict inequality, suppose, by contradiction, that $\Gamma_{i} f\left(w_{b}, \zeta\right)=0$ for some $\zeta \in \mathcal{Z}$. This would require $\Gamma_{i 2} B_{2}( \pm \eta-\zeta)=0$, which is impossible, since $\zeta \leq \bar{\zeta}<\eta$. Thus, $w_{b}$ is a point satisfying the Lemma. The case when $\partial G_{i} \cap \mathcal{H}_{\mathcal{S}_{o}}\left[\underline{w}_{2}, \bar{w}_{2}\right] \cap w_{2}^{+}=\emptyset$ is treated similarly, taking $b=\max (V)$, which is negative, and considering the cylinder $\mathcal{H}_{\mathcal{S}_{o}}\left[b, \bar{w}_{2}\right]$. Finally, consider the remaining possibilities of $\partial G_{i} \cap \mathcal{H}_{\mathcal{S}_{o}}\left[\underline{w}_{2}, \bar{w}_{2}\right] \cap w_{2}^{o} \neq \emptyset$ or $\Gamma_{i 2}=0$. In those cases, the intersection of $\partial G_{i}$ with the cylinder and the sliding hyperplane $w_{2}=0$ is nonempty. System dynamics on the sliding hyperplane is given by $\dot{w}_{1}=A_{w} w_{1}$. Consider the set resulting from the intersection of $\mathcal{S}_{o}$ and the closure of the complement of the state constraint restricted to $w_{2}=0$, that is, let $K=\mathcal{S}_{o} \cap \overline{G_{o i}^{c}}$, where $G_{o i}^{c}=\left\{w_{1} \in \mathbb{R}^{n-1}: \Gamma_{i 1} w_{1}>1\right\}$. It is easy to see that $K$ is compact and convex and that it does not contain the origin. Suppose, by contradiction, that $\Gamma_{i 1} \dot{w}_{1} \geq 0 \forall w_{1} \in \partial G_{o i} \cap \mathcal{S}_{o}$. Then, following arguments similar to those used in the proof of Theorem 4, it is possible to deduce that $K$ is positively invariant for $\dot{w}_{1}=A_{w} w_{1}$, which contradicts the asymptotic stability of the origin. Thus, $\exists w_{1} \in \partial G_{o i} \cap \mathcal{S}_{o}$ such that $\Gamma_{i 1} \dot{w}_{1}<0$, which in turns implies that $w=\left[w_{1}^{T} \mid 0\right] \in \partial G_{i} \cap \mathcal{H}_{\mathcal{S}_{o}}\left[\underline{w}_{2}, \bar{w}_{2}\right]$ satisfies $\Gamma_{i} \dot{w}<0$.

\section{APPENDIX IV \\ PROOF OF COROLLARY 1}

By hypothesis, and using Proposition 1 , we have that either $\mathcal{H}_{\mathcal{S}_{o}}\left[\underline{w}_{2}, \bar{w}_{2}\right] \cap \partial G_{i} \quad \subset \operatorname{int}\left(G_{i}^{+}\right)$or $\mathcal{H}_{\mathcal{S}_{o}}\left[\underline{w}_{2}, \bar{w}_{2}\right] \cap$ $\partial G_{i} \subset \operatorname{int}\left(G_{i}^{-}\right)$. For the first possibility, we have that

$$
\mathcal{H}_{\mathcal{S}_{o}}\left[\underline{w}_{2}, \bar{w}_{2}\right] \cap \partial G_{i} \subset \operatorname{int}\left(G_{i}^{+}\right) \subset G_{i}^{+} .
$$

Then, for all $w \in \mathcal{H}_{\mathcal{S}_{o}}\left[\underline{w}_{2}, \bar{w}_{2}\right] \cap \partial G_{i}, \exists \zeta \in \mathcal{Z}$ such that $\Gamma_{i} f(w, \zeta)>0$, contradicting Lemma 3 . Therefore, it must be true that $\mathcal{H}_{\mathcal{S}_{o}}\left[\underline{w}_{2}, \bar{w}_{2}\right] \cap \partial G_{i} \subset \operatorname{int}\left(G_{i}^{-}\right)$. However, $\operatorname{int}\left(G_{i}^{-}\right) \cap$ $G_{i}^{+}=\varnothing$, therefore $\mathcal{H}_{\mathcal{S}_{o}}\left[\underline{w}_{2}, \bar{w}_{2}\right] \cap \partial G_{i} \cap G_{i}^{+}=\varnothing$. Finally, since $\mathcal{S} \subset \mathcal{H}_{\mathcal{S}_{o}}\left[\underline{w}_{2}, \bar{w}_{2}\right]$, we have $\mathcal{S} \cap \partial G_{i} \cap G_{i}^{+}=\varnothing$. The Corollary now follows from Theorem 4 .

\section{REFERENCES}

[1] U. Itkis, Control Systems of Variable Structure. New York: Wiley, 1977.

[2] M. Jackson and Y. Shtessel, "Sliding mode thermal control system for space station furnace facility," IEEE Trans. Control Syst. Technol., vol. CST-6, no. 5, pp. 612-622, Sep. 1988.

[3] S. Ouenou-Gamo et al., "A nonlinear controller of a turbocharged diesel engine using sliding mode," in Proc. IEEE Int. Conf. Control Appl., 1997, pp. 803-805.

[4] J. Slotine and W. Li, Applied Nonlinear Control. Englewood Cliffs, NJ: Prentice-Hall, 1991.

[5] V. Utkin, "Variable structure systems with sliding modes: A survey," IEEE Trans. Autom. Control, vol. AC-22, pp. 212-222, 1977.

[6] V. Utkin, Sliding Modes in Control Optimization. New York: Springer-Verlag, 1992.

[7] F. Blanchini, "Set invariance in control," Automatica, vol. 35, pp. 1747-1767, 1999.

[8] B. O'Dell and E. Misawa, "Semi-ellipsoidal controlled invariant sets for constrained linear systems," ASMS J. Dyn. Syst. Meas. Control, vol. 124, pp. 98-103, 2002.

[9] P. Keleher and R. Stonier, "Adaptive terminal sliding mode control of a rigid manipulator with uncertain dynamics incorporating state constraints," Australian and New Zealand Industrial Appl. Math. J., vol. 43E, pp. E102-E152, 2002.

[10] M. Innocenti and M. Falorni, "State constrained sliding mode controllers," in Amer. Control Conf., 1998, pp. 104-108.

[11] M. Nagumo, "Über die lage der integralkurven gewöhnlicher differen-tialgleichungen," Proc. Physico-Math. Soc. Japan, vol. 24, pp. 272-559, 1942.

[12] J. Aubin and A. Cellina, Differential Inclusions. New York: SpringerVerlag, 1988.

[13] V. Krivan, "On the intersection of contingent cones," J. Optim. Theory Appl., vol. 70, no. 2, pp. 397-404, 1991.

[14] C. Edwards and S. Spurgeon, Sliding Mode Control. Englewood Cliffs, NJ: Prentice-Hall, 1990.

[15] A. Filippov, Differential Equations with Discontinuous Righthand Sides. Norwell, MA: Kluwer, 1988. 
[16] H. Richter, "Theoretical tools and software for modeling, simulation and control design of rocket test facilities," NASA, Stennis Space Center, MS, Tech. Memorandum SSTI-8060-0001, 2004 [Online]. Available: http://ntrs.nasa.gov

[17] B. O'Dell, "Ellipsoidal and semiellipsoidal controlled invariant sets for constrained linear systems" Ph.D. dissertation, Dept. Mech. Aerosp. Eng., Oklahoma State Univ., Stillwater, OK, 1999 [Online]. Available: http://acl.okstate.edu/ach-eports.html

[18] M. Brockman and M. Corless, "Quadratic boundedness of nominally linear systems," Int. J. Control, vol. 71, no. 6, pp. 1105-1117, 1998.

[19] J. Hung and J. Hung, "Chatter reduction in variable-structure control," Proc. 20th IEEE Industrial Electronics Society Conference (IECON), vol. 3, pp. 1914-1918, 1994.

[20] M. Hamerlain et al., "Switching on the derivative of control to reduce chatter," Inst. Electr. Eng. Proc.-Control Theory Appl., vol. 148, no. 1, pp. 88-96, 2001.

[21] T. Kailath, Linear Systems. Englewood Cliffs, NJ: Prentice-Hall, 1980.

[22] A. Michel and C. Herget, Applied Algebra and Functional Analysis. New York: Dover, 1981. 